\title{
Three-dimensional visualization of arsenic stimulated mouse liver sinusoidal by FIB-SEM approach
}

\section{Dear Editor,}

Liver sinusoidal endothelial cells (LSECs) are the most abundant non-parenchymal and highly specialized fenestrated cells in the liver. LSECs differ from endothelial cells of other capillaries due to the presence of open fenestrations and the absence of a basal lamina (Wisse et al., 1996; Braet and Wisse, 2002; Aird, 2007; Cogger et al., 2008). During the differentiation process, LSECs become fenestrated to facilitate the transfer of circulating nutrients, lipids, and lipoproteins between blood and the space of Disse for normal liver metabolism. Only particles smaller than the fenestrae can reach the hepatocytes or leave the space of Disse (Wisse et al., 1985). The size of fenestration changes upon different conditions to regulate the substrate exchange.

Previous studies reported that the diameter of the sinusoidal fenestrations varies between different species from $107 \pm 1.5 \mathrm{~nm}$ (mean \pm SEM) in the human liver (Wisse et al., 2008 ) to $141 \pm 5.4 \mathrm{~nm}$ (mean \pm SEM) in the mouse liver (Wisse et al., 2008), which was determined based on transmission electron micrographs of sections (Jacobs et al., 2010). However, considering the small size of fenestration, $100 \sim 150 \mathrm{~nm}$ in diameter, which is beyond the limit of resolution in light microscopy (Cogger and Couteur, 2009), in early stage, the morphology of fenestrations could only be studied primarily using conventional electron microscopy (Owen et al., 2010; Svistounov et al., 2012), however, with lack of 3D information.

Arsenic is a toxic metalloid and a common contaminant of drinking water. Drinking arsenic-contaminated water increases the risk of cardiovascular disease, lung disease, hepatic disease, and cancer in millions of people worldwide. Even at low levels, arsenic promotes angiogenesis and vascular remodeling in mice (Guha Mazumder, 2003; Mazumder, 2005; Navas-Acien et al., 2005). Pathological vascular remodeling, such as neovascularization, angiogenesis, and morphologic changes in vascular architecture, is a critical process in the development of vascular diseases including atherosclerosis, cardiovascular ischemic diseases, tumor vasculogenesis, and liver fibrosis. Different from angiogenesis of systemic vessels endothelial cells, LSECs angiogenesis, also called capillarization, is a dedifferentiation and maturation process with diagnostic hallmarks of LSEC defenestration and renewed surface expression of PECAM-1 and laminin-1 in response to environmental stress and aging (Straub et al., 2007). Liver capillarization precedes vascular remodeling of other liver vessels, such as hepatic arterioles and PBVP (peribiliary vascular plexus), causing blood flow shunting, vascular channel formation, and eventually liver fibrosis (Straub et al., 2007). Liver capillarization also affects the systemic vasculature and promotes atherogenesis by decreasing liver metabolism of lipids, lipoproteins, and glucose (Straub et al., 2007).

In this study, we investigated how the fenestration morphology of LSECs in mice changes in response to an arsenic stimulation. We utilized focused ion beam scanning electron microscopy (FIB-SEM) technique to reconstruct the $3 \mathrm{D}$ morphology $\left(20 \times 20 \times 1.5 \mu^{3}\right)$ of mouse liver sinusoidal with and without the arsenic stimulation. The three-dimensional data generated from FIB-SEM approach enabled us to perform morphologic comparison in 3D space and quantitative measurements and comparisons for the size and number of LSEC fenestrations.

FIB-SEM is a technique to generate high resolution threedimensional images of biological samples in micrometer scale (Kizilyaprak et al., 2014). Samples are prepared by a similar method to transmission electron microscopy, typically by fixing the sample with aldehyde, staining with heavy metals such as osmium and uranium then embedding in an epoxy resin. The surface of the block of resin-embedded sample is imaged by detection of back-scattered electrons. Following imaging, the focused ion beam is used to trim a thin section (typically less than $30 \mathrm{~nm}$ ) from the face of the block. After the section is trimmed, the sample block is raised back to the focal plane and imaged again. This sequence of sample imaging, section trimming and block raising can acquire many thousands of images with perfect alignment in an automated fashion and yield a $3 \mathrm{D}$ volume data of specimen.

The livers from both normal and As(III)-exposed mice (see Supplemental Materials and Methods) were firstly fixed, embedded in resin and sectioned for conventional transmission 
electron microscopy (TEM) examination. The region of the sinusoidal endothelium was selected and imaged. In consistency with the previous observation (Straub et al., 2007), the LSECs in normal mice contain numerous sieve plates with open fenestrae (Fig. 1A). While, the liver sinusoids in the As(III)-exposed mice become defenestrated with continuous endothelium membrane (Fig. 1B). Besides, the density of hepatocyte microvilli in the space of Disse, which protrude through the LSEC fenestrae, becomes significantly increased in the As(III)-exposed liver sinusoids (Fig. 1B).

Since the size of fenestrations $(>100 \mathrm{~nm})$ is larger than the thickness of normal TEM sections $(\sim 70 \mathrm{~nm})$, the overall morphology of fenestrations and their distribution along the sinusoidal vessel could not be resolved from a single TEM image. To get a more accurate observation of mouse liver sinusoidal fenestrations, we utilized focused ion beam scanning electron microscopy (FIB-SEM) technique to reconstruct the $3 \mathrm{D}$ morphology of mouse liver sinusoid in micrometer scale.

The SEM images of both normal and As(III)-exposed mouse liver sinusoid show perfect contrast and similar morphology observation (Fig. 1C and 1D) to that of TEM images (Fig. 1A and 1B). We collected 116 sequential FIBSEM images $(4096 \times 4096)$ with the pixel size of $5 \mathrm{~nm}$ and the milling thickness of $15 \mathrm{~nm}$ to cover $\sim 20 \times 20 \times 1.5 \mu \mathrm{m}^{3}$ size of specimen for both normal and As(III)-exposed mouse liver sinusoid (Movies S1 and S2), respectively. After image alignment, denoising and reconstruction (see Supplemental Materials and Methods), the 3D morphology of mouse liver sinusoid was obtained (Fig. 1E and $1 \mathrm{~F}$ ), showing the morphology of LSECs and their fenestrations clearly in 3D space. Besides, the hepatocyte microvilli in the space of Disse are also well resolved. The small deformation factors for the rotation, magnification, and stretch corrections (Figs. $2 \mathrm{G}, 2 \mathrm{H}$, and $\mathrm{S} 1$ ), which are less than $6 \%, 1 \%$, and $1 \%$, respectively, demonstrate the data collection stability of the FIB-SEM method and confirm the high quality of the 3D reconstructions.

At the current experiments, the pixel size of each FIBSEM image is about $5 \mathrm{~nm}$ in XY direction and the FIB cutting step size is $15 \mathrm{~nm}$ in $Z$ direction, and the size of live sinusoid fenestrations are approximately $50-150 \mathrm{~nm}$ in diameter, thus the fenestrations can be well resolved after the reconstructions by FIB-SEM method (Fig. 2A and 2B). In normal mice, most fenestrations are irregular holes with a relative large size (Fig. 2A) while, in As(III)-exposed mice, a majority of fenestrations appear as approximately round holes and most of them are relatively smaller than those in normal ones (Fig. 2B).

Based on the 3D morphologies of the liver sinusoids (Fig. 1E and 1F), we were able to count the number of fenestrations and measure their sizes statistically. Since the fenestration is irregular, we utilized a minimal rectangle to cover each fenestration and used the area of that rectangle as the approximate determinant of the fenestration size. From the statistic histogram of the numbers of fenestrations
Figure 1. Visualization of mouse liver sinusoidal by TEM and 3D reconstruction of mouse liver sinusoid by FIB-SEM. (A) TEM micrograph of sinusoid in normal mice, which contain numerous sieve plates with open fenestrae. (B) TEM micrograph of sinusoid in arsenic treated mice, which show defenestration with continuous endothelium. The boxed region is zoomed in at the bottom. EC, endothelial cell; $D$, Disse; $M$, microvilli; $V$, sinusoidal vessel. (C and $D)$ SEM micrographs of sinusoid in normal mice and arsenic treated mice respectively. (E and F) FIBSEM reconstructions of sinusoid in normal mice and arsenic treated mice respectively. The scales in $X, Y$, and $Z$ directions are labeled with the pixel size of $5 \mathrm{~nm}$ for $X Y$ direction and $15 \mathrm{~nm}$ for $Z$ direction. ( $G$ and $H$ ) The statistic diagrams of the parameters including the image rotation angle $(X$ axis), the image magnification correction factor $(Y$ axis) and the image stretch correction factor ( $Z$ axis) during image alignment and deformation correction of serial SEM images of sinusoid in normal mice $(G)$ and arsenic treated mice $(H)$ respectively. These parameters are calculated from two sequential adjacent images (see also Fig. S1).

vs. their sizes, we could observe the different distributions of the fenestrations for the normal and As(III)-exposed specimen. For the normal specimen, the size distribution of the fenestrations is relatively uniform, while, for the As(III)-exposed specimen, more fenestrations tend to have a smaller size (Fig. 2C). We further counted the percentages of fenestrations in different sizes. For the normal specimen, the percentage of fenestrations with the size smaller than $15,000 \mathrm{~nm}^{2}$ is $50 \%$ and $62 \%$ for the size smaller than $20,000 \mathrm{~nm}^{2}$ (Fig. 2D). However, for the As(III)-exposed specimen, the percentage of fenestrations with the size smaller than $15,000 \mathrm{~nm}^{2}$ is increased to $66 \%$ and $77 \%$ for the size smaller than $20,000 \mathrm{~nm}^{2}$ (Fig. 2D). In total, the average area of fenestrations is about $18,000 \mathrm{~nm}^{2}$ for the normal specimen and about $10,000 \mathrm{~nm}^{2}$ for the As(III)-exposed one. As the result, the above quantitative analysis of the 3D morphologies of the liver sinusoids clearly reveals that the liver sinusoids become defenestrated upon As (III)-exposure.

The thicknesses of the epithelia layers are visually different between normal and As(III)-exposed specimen according to both TEM (Fig. $1 \mathrm{~A}$ and $1 \mathrm{~B}$ ) and FIB-SEM (Fig. 2E and 2F) analyzes. We further measured the averaged thicknesses of the epithelia layers quantitatively and got the measurements of $200 \mathrm{~nm}$ and $270 \mathrm{~nm}$ for the normal and As(III)-exposed specimen, respectively (Fig. 2G). As a result, these comparisons suggest that after As(III)-exposure the thickness of the epithelia layers of live sinusoids is increased.

FIB-SEM has been recently emerging as an efficient technique to study the 3D high-resolution ultrastructure of cells and tissues by acquiring thousands of sequential surface images automatically in a perfect alignment. Here, we utilized FIB-SEM technique to achieve 3D reconstruction of 

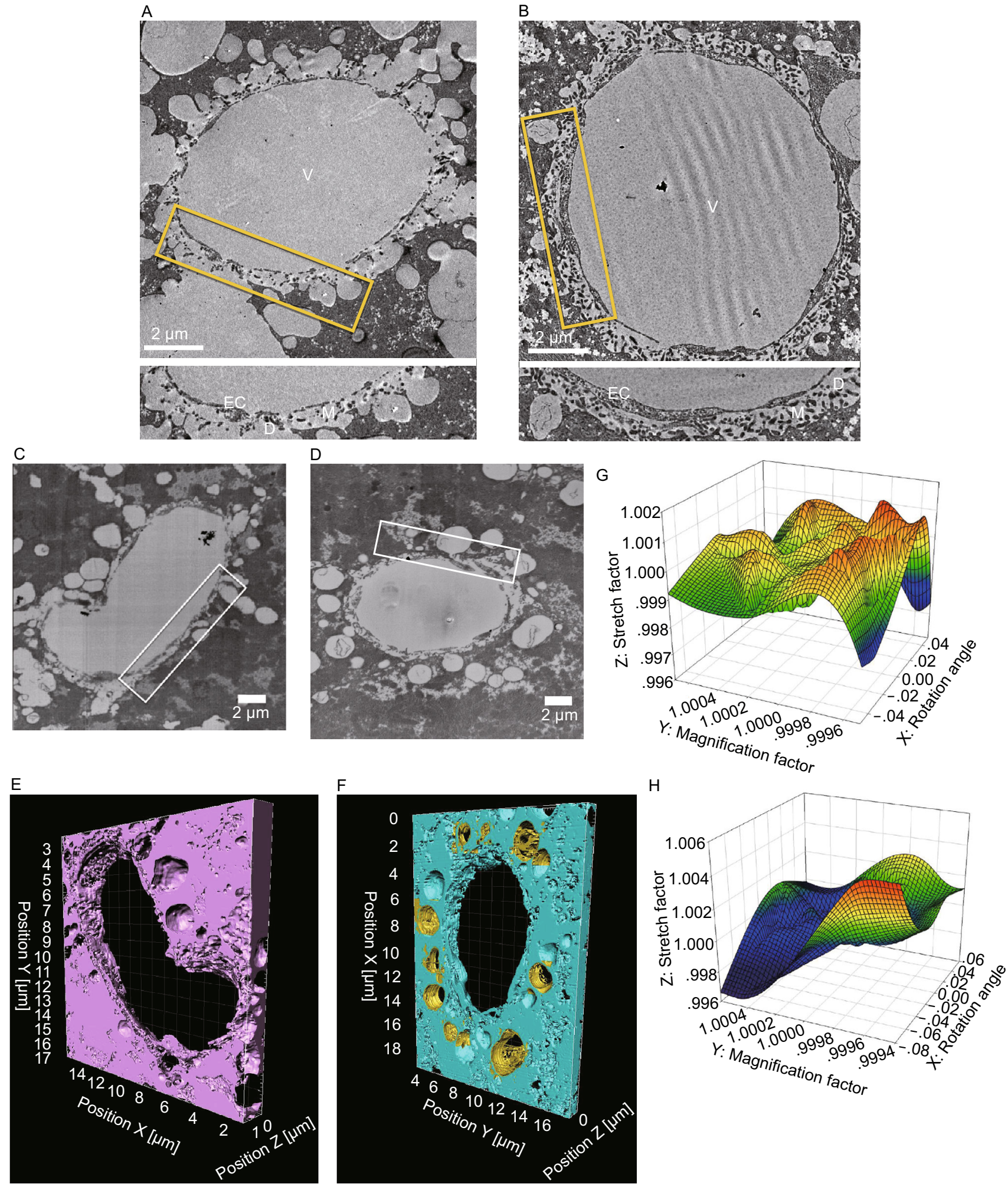
A

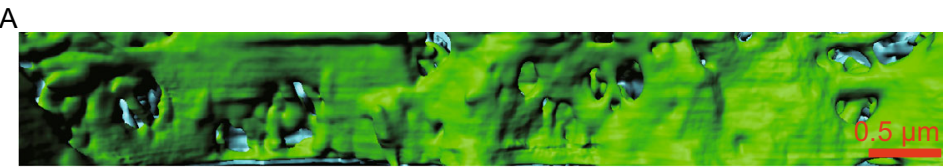

B

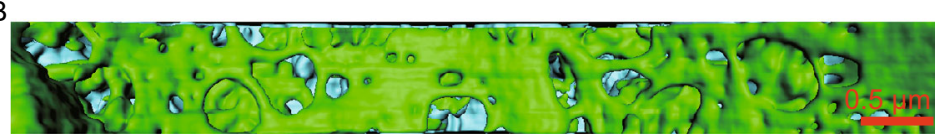

C

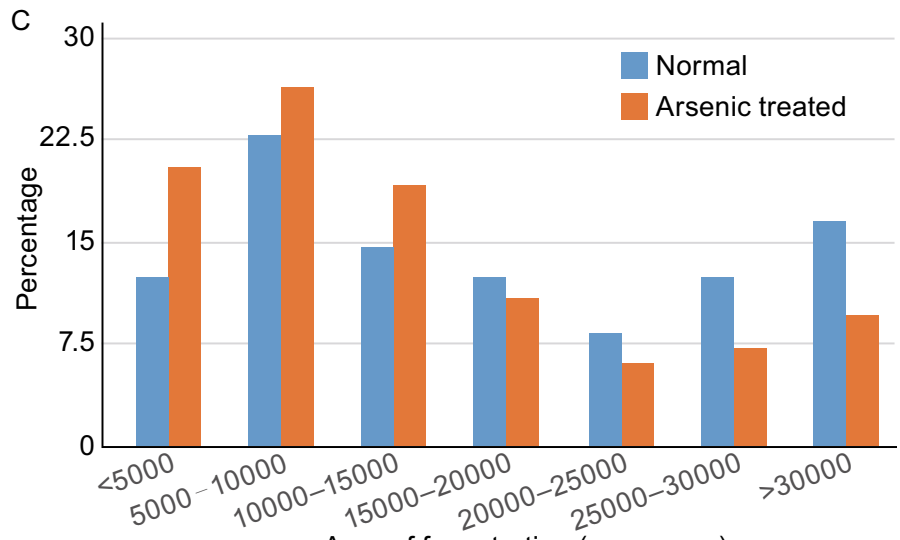

Area of fenestration (square $\mathrm{nm}$ )

D

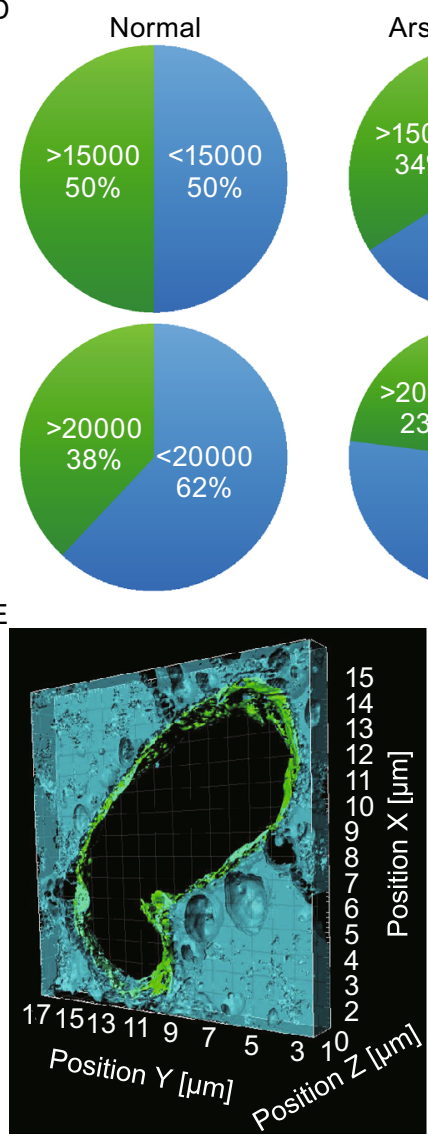

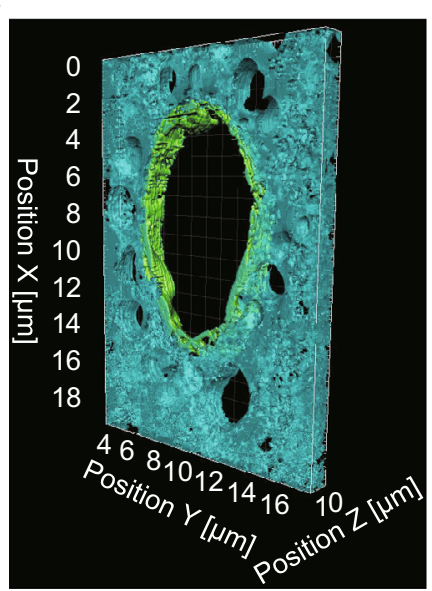

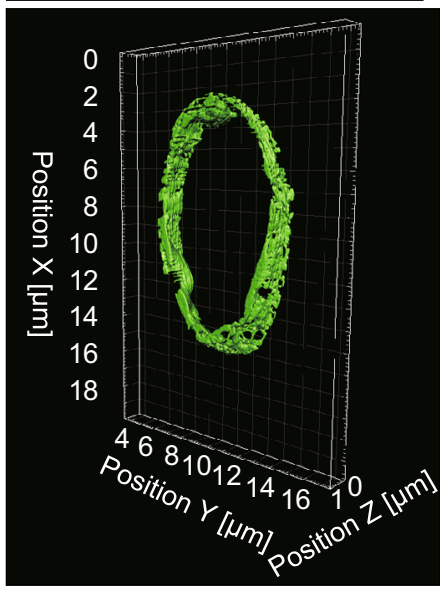

G

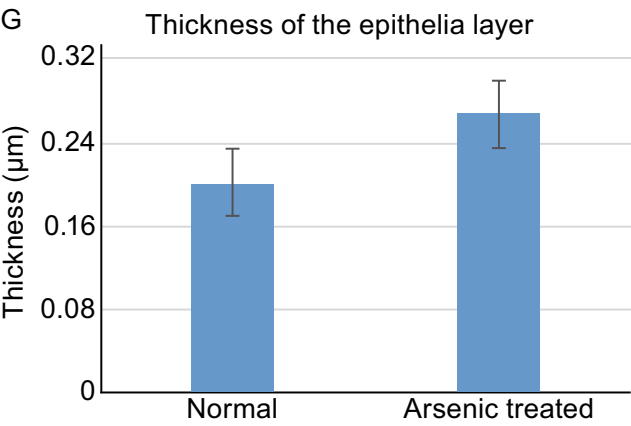


Figure 2. 3D Morphology comparison and quantitative analysis of fenestrations and epithelial cells layers between normal and arsenic treated specimen. (A and $B$ ) 3D morphology of fenestrations in normal mice and arsenic treated mice respectively. The endothelial cells are colored in green and the hepatocyte microvilli are colored in cyan. (C) The histogram statistics of the numbers of fenestrations vs. their areas in normal mice (blue) and arsenic treated mice (orange) respectively. The numbers of fenestrations are normalized and shown in their percentages. (D) The statistics of the fenestration numbers larger or smaller than $15,000 \mathrm{~nm}^{2}$ (up) or $20,000 \mathrm{~nm}^{2}$ (bottom) for normal specimen (left) and arsenic treated specimen (right). ( $E$ and $F$ ) 3D morphology of sinusoid in normal mice $(E)$ and arsenic treated mice $(F)$ with the epithelia layer colored in green and segmented in right. The scales in $X, Y$, and $Z$ directions are labeled with the pixel size of $5 \mathrm{~nm}$ for $X Y$ direction and $15 \mathrm{~nm}$ for $Z$ direction. (G) Comparison of the averaged thickness of the epithelial layers for the normal mice and arsenic treated mice. See Supplemental Materials and Methods for the details of epithelial layer thickness measurements, sampling, and averaging.

mouse liver SEC ultrastructure within $1.5 \mu \mathrm{m}$ scale and 15 $\mathrm{nm}$ step size in $Z$ direction and $5 \mathrm{~nm}$ pixel size in $X Y$ plane, and observed the ultra-structural changes upon the arsenic stimulation. By comparing the normal specimen with the As (III)-exposed one in three-dimensional space, we found that after As(III)-exposure, the sizes of live sinusoid fenestrations are reduced and the thicknesses of the epithelia layers are increased. Our present results provided a further support to understand the molecular mechanism of LSECs regulation and established a state-of-the-art approach to investigate LSECs in a large scale.

\section{FOOTNOTES}

This work was supported by grants from the Strategic Priority Research Program of Chinese Academy of Sciences (XDB08030202) and the National Basic Research Program (973 Program) (No. 2014CB910700). All the EM works were performed at Center for Biological Imaging (CBI, http://cbi.ibp.ac.cn), Institute of Biophysics, Chinese Academy of Sciences.

WL and FS designed research; WL and LW performed sample preparation; GJ and JZ collected EM data; WD performed image processing and 3D reconstruction; FS and WD analyzed data; FS, WL, and WD wrote the paper with the input from other authors.

All the authors declare that they have no conflict of interest. All institutional and national guidelines for the care and use of laboratory animals were followed.

Wenbo Li and Wei Ding have equal contributions to this work.

Electronic supplementary material The online version of this article (doi:10.1007/s13238-016-0246-9) contains supplementary material, which is available to authorized users.
Wenbo $\mathrm{Li}^{1}$, Wei Ding ${ }^{2}$, Gang Ji ${ }^{2}$, Li Wang ${ }^{2}$, Jianguo Zhang ${ }^{2}$, Fei Sun ${ }^{1,2,3 \bowtie}$

${ }^{1}$ National Laboratory of Biomacromolecules, Institute of Biophysics, Chinese Academy of Sciences, Beijing 100101, China

${ }^{2}$ Center for Biological Imaging, Institute of Biophysics, Chinese Academy of Sciences, Beijing 100101, China

${ }^{3}$ College of Life Science, University of Chinese Academy of Sciences, Beijing 100049, China

$\checkmark$ Correspondence: feisun@ibp.ac.cn (F. Sun)

\section{OPEN ACCESS}

This article is distributed under the terms of the Creative Commons Attribution 4.0 International License (http://creativecommons.org/ licenses/by/4.0/), which permits unrestricted use, distribution, and reproduction in any medium, provided you give appropriate credit to the original author(s) and the source, provide a link to the Creative Commons license, and indicate if changes were made.

\section{REFERENCES}

Aird WC (2007) Phenotypic heterogeneity of the endothelium: II. Representative vascular beds. Circ Res 100:174-190

Braet F, Wisse E (2002) Structural and functional aspects of liver sinusoidal endothelial cell fenestrae: a review. Comp Hepatol 1:1

Cogger VC, Couteur DGL (2009) Fenestrations in the liver sinusoidal endothelial cell. Wiley, Hokoben

Cogger VC, Arias IM, Warren A, Mcmahon AC, Kiss DL, Avery VM, Le CD (2008) The response of fenestrations, actin, and caveolin1 to vascular endothelial growth factor in SK Hep1 cells. Am J Physiol Gastrointest Liver Physiol 295:G137-G145

Guha Mazumder DN (2003) Chronic arsenic toxicity: clinical features, epidemiology, and treatment: experience in West Bengal. J Environ Sci Health Part A 38:141-163

Jacobs F, Wisse E, De Geest B (2010) The role of liver sinusoidal cells in hepatocyte-directed gene transfer. Am J Pathol 176:14-21

Kizilyaprak C, Daraspe J, Humbel BM (2014) Focused ion beam scanning electron microscopy in biology. J Microsc 254:109-114

Mazumder DG (2005) Effect of chronic intake of arsenic-contaminated water on liver. Toxicol Appl Pharmacol 206:169-175

Navas-Acien A, Sharrett AR, Silbergeld EK, Schwartz BS, Nachman KE, Burke TA, Guallar E (2005) Arsenic exposure and cardiovascular disease: a systematic review of the epidemiologic evidence. Am J Epidemiol 162:1037-1049

Owen DM, Gaus K, Magee Al, Cebecauer M (2010) Dynamic organization of lymphocyte plasma membrane: lessons from advanced imaging methods. Immunology 131:1-8

Straub AC, Stolz DB, Ross MA, Hernández-Zavala A, Soucy NV, Klei LR, Barchowsky A (2007) Arsenic stimulates sinusoidal endothelial cell capillarization and vessel remodeling in mouse liver. Hepatology 45:205-212

Svistounov D, Warren A, McNerney GP, Owen DM, Zencak D, Zykova SN, Crane H, Huser T, Quinn RJ, Smedsrød B (2012) The relationship between fenestrations, sieve plates and rafts in liver sinusoidal endothelial cells. PLoS One 7:e46134

Wisse E, De Zanger R, Charels K, Van Der Smissen P, McCuskey R (1985) The liver sieve: considerations concerning the structure 
and function of endothelial fenestrae, the sinusoidal wall and the space of Disse. Hepatology 5:683-692

Wisse E, Braet F, Luo D, De Zanger R, Jans D, Crabbe E, Vermoesen A (1996) Structure and function of sinusoidal lining cells in the liver. Toxicol Pathol 24:100-111
Wisse E, Jacobs F, Topal B, Frederik P, De Geest B (2008) The size of endothelial fenestrae in human liver sinusoids: implications for hepatocyte-directed gene transfer. Gene Ther 15:1193-1199 